\title{
The Role of Sense of Coherence and Personal Intelligence in Prediction the Craving of Substance Abusers
}

Hajloo. N1

Ahmadi. $\mathrm{SH}^{2}$

${ }^{*}$ Gharibzadeh. $\mathrm{R}^{3}$

1- Ph.D. Psychology, Professor, Psychology Department, Faculty of Educational Sciences and Psychology, University of Mohaghegh Ardabili, Ardabil, Iran.

2- Ph.D. Students in Psychology, Psychology Department, Faculty of Educational Sciences and Psychology, University of Mohaghegh Ardabili, Ardabil, Iran.

3- ( ${ }^{\star}$ Corresponding Author) Ph.D. Students in Educational Administration, Educational Sciences Department, Faculty of Educational Sciences and Psychology, University of MohagheghArdabili, Ardabil, Iran.

Email: ramingharibzadeh71@ gmail.com

\section{Abstract}

Introduction: Drug abuse has been affected by a combination of individual, family and social factors. Craving for drug abuse has been recognized as a predictor of negative outcomes of treatment of drug abuse disorders.

Objective: The present study aimed to determine the role of a sense of coherence and personal intelligence in the prediction of the craving of drug users

Material and Methods: The present study is descriptive and correlational. The population of the study consisted of all drug users who referred to treatment centers in the first half of 2018 in the outpatient departments of Kermanshah province, a total of 120 individuals were selected through cluster random sampling. Data collection tools consisted of three questionnaires including a sense of coherence, personal intelligence and craving. Data were analyzed by descriptive statistics, Pearson correlation coefficient and multiple regressions using SPSS 25

Results: The results of correlation coefficients showed that the craving had a negative and significant relationship with the total score of sense of coherence, that of the components of Comprehensibility, Controllability, Significance and Configuration, meaningful and shaping $(\mathrm{P}<0.01)$. The results of the regression analysis showed that 30 percent of craving variance was explained by a sense of coherence, and personal intelligence.

Discussion and Conclusion: Accordingly, the sense of coherence and personal intelligence is one of the variables related to craving that can be targeted for the prevention, treatment and depression of these variables.

Keywords: Craving, Drug Users, Intelligence, Sense of Coherence. 


\title{
نقش حس انسجام و هوش شخصى در بيشبينى ولع افراد مصرفكننده مواد
}

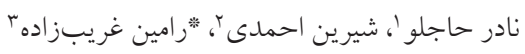

جكيده

مقدمه: مصرف مواد تحت تأثير مجموعهاى از عوامل فردى، خانوادگى و اجتماعى قرار دارد و ولع مصرف به عنوان ييشبينى كنندهى بيامدهاى منفى درمان در برطرف كردن درمان اختلال هاى مصرف مواد مخدر شناخته شده است.

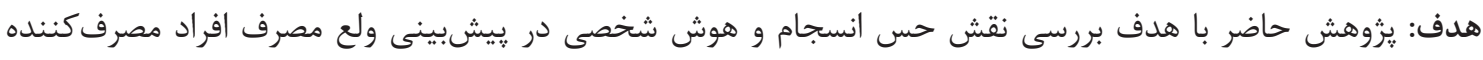
مواد انجام گرفت. مواد و روشها: يزوهش حاضر از نوع توصيفى - همبستكى صورت كرفته است. كليه افراد مصرف كننده مواد كه در

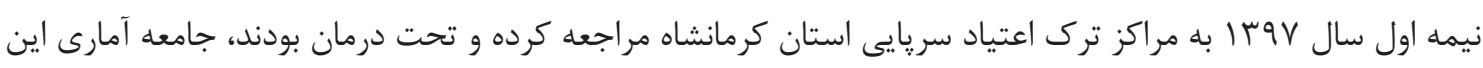

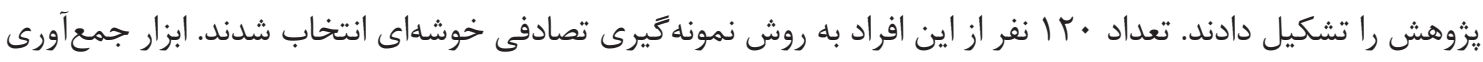

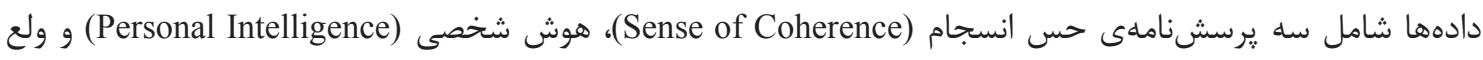

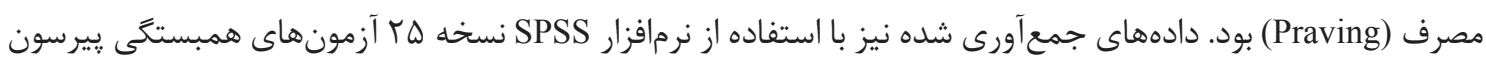
و تحليل ركرسيون خند كانه در سطح خطاى هـ • • تحليل شد.

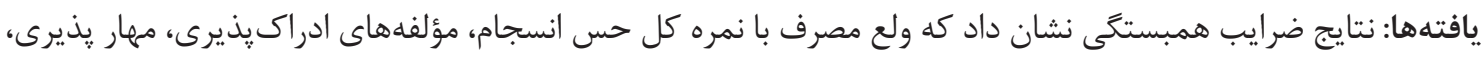

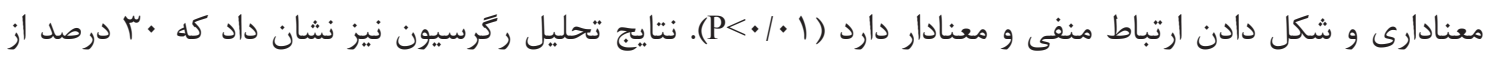

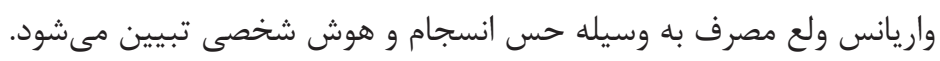

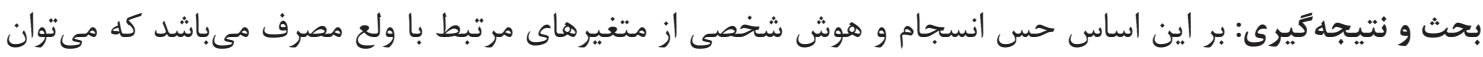

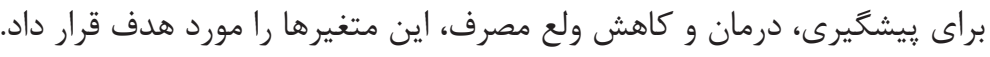

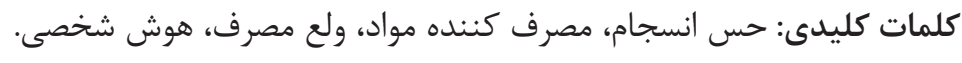

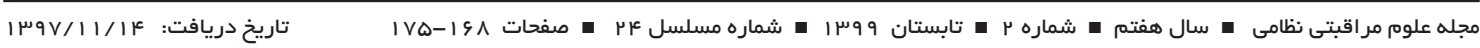

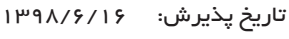

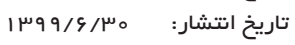

سلامت و كيفيت رفتارى بيشتر از ب ميليون و ^•^ هزار نفر در مقلمه

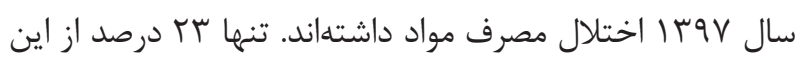
بيمارى اعتياد يك بيمارى اصلى، مزمن و عصب زيستشناسى

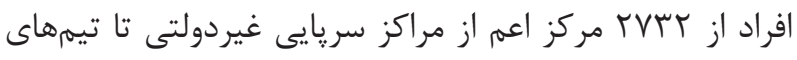

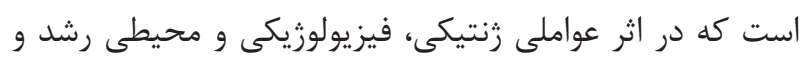
درمانهاى اجتماع محور درمان دريافت مىكنند (بّ). بروز يِدا مى كند و وجه مشخصه آن اختلال در كنترل انجام

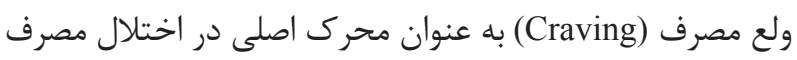

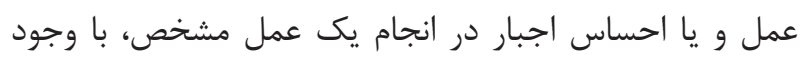

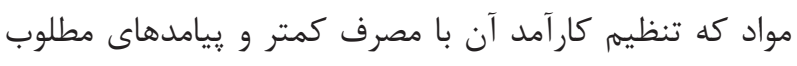
آكاهى نسبت به عواقب خطرناك آن است (1). اعتياد به مواد تداعى شده است محسوب مىشود (أ). ولع مصرف به عنوان

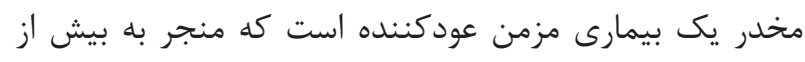

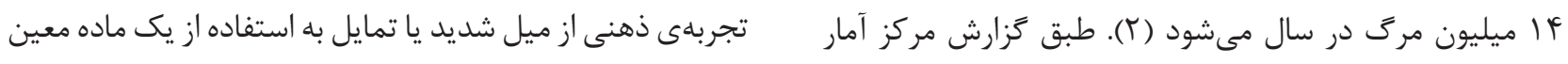


اطلاعات مرتبط با شخصيت و نيز در احساس درد و لذت كاربرد

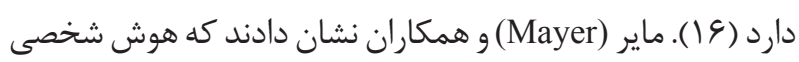

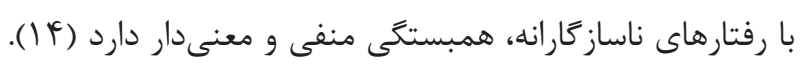

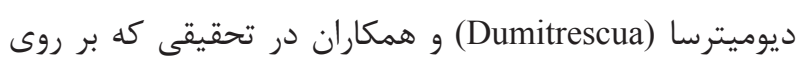

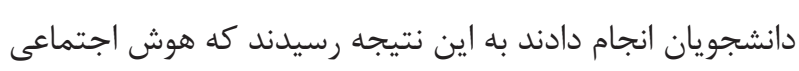

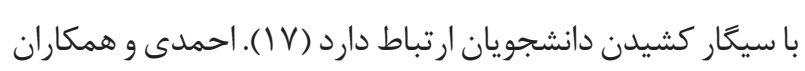

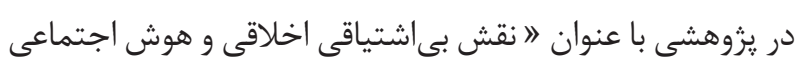

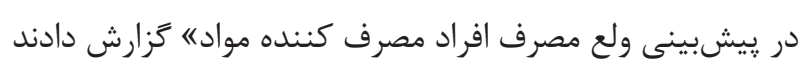
مصرف مواد و ولع مصرف با هوش اجتماعى ارتباط منفى دارد

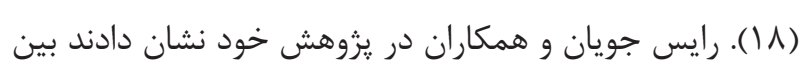

هوش هيجانى و بازَشت به اعتياد ارتباط وجود دارد (19 (1). توجه جامعه علمى به نقش ولع مصرف در اعتياد، منعكس كنندهى معرفى ولع مصرف به عنوان عامل تشخيصى مهرم در تعريف

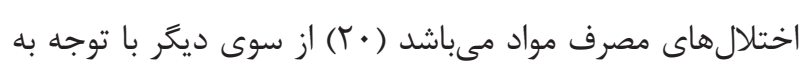

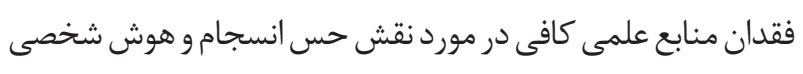
در ولع مصرف و همجنين با در نظر كرفتن اينكه در كشور ما در حال حاضر به بررسى نقش اين متغير ها در ولع مصرف مواد يرداخته نشده

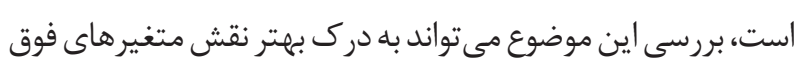

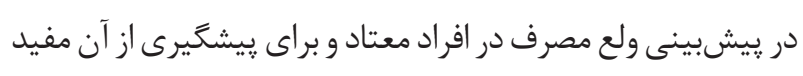

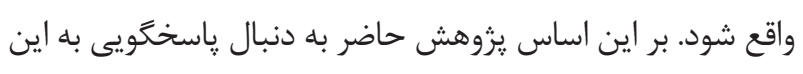
سؤال است كه آيا حس انسجام و هوش شخصى توانايى بيش بيش بينى ولع مصرف افراد مصرف كننده مواد را دارند؟ أنج هوش شيخ

\section{مواد و روشها}

يزوهش حاضر توصيفى و از نوع مطالعات همبستخى صورت كرفته است. جامعه آمارى يزوهش حاضر را كليه افراد مصرف كننده مواد مر اجعه كننده به مراكز ترك اعتياد استان كرمانشاه تشكيل دادند.

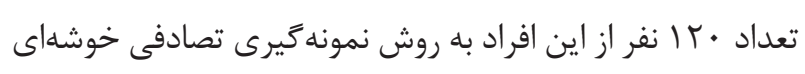

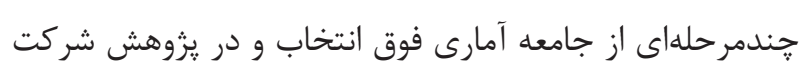

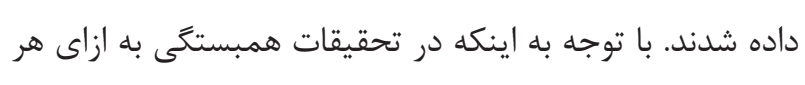

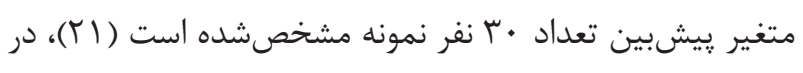

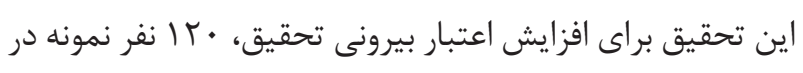

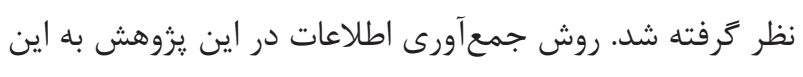
صورت بود كه ابتدا ليست تمامى مراكز ترك اعتياد استان كرمانشاه
و شدت اشتياق فرد در هنگام تلاش براى تغيير تعريف شده است

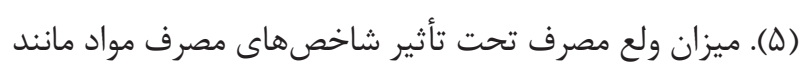



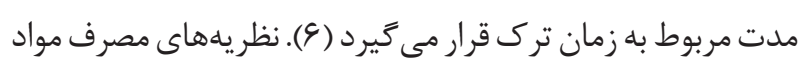
بر نقش ولع مصرف در استفاده مداوم مواد مخدر تأكيد دارند (V).

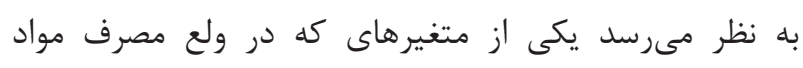

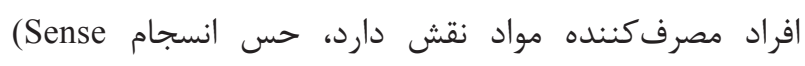
است. حس انسجام توسط آنتونووسكى (A) of Coherence) مطرح شد (^). وى حس انسجام را به صورت (Antonovksy) يكى جهت كيرى شخصى به زندىى تعريف مى كند او بر اين باور

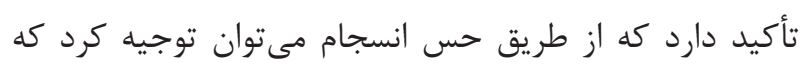

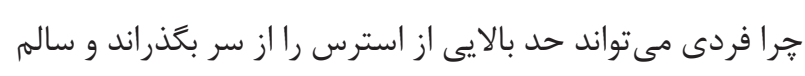

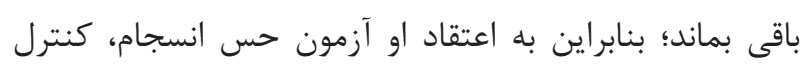

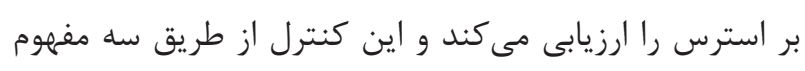

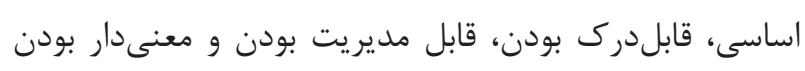

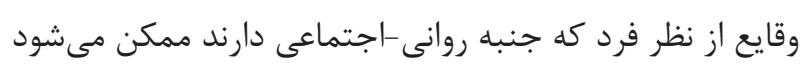

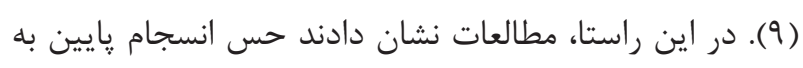
صورت قابل توجه با مصرف خطرناك الكل مرتبط است ( • (). در يزوهشى كه ارغابايى (Arghabaei) و همكاران انجام دادند

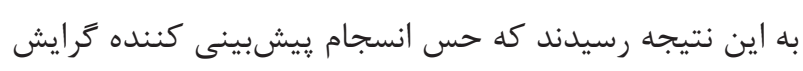

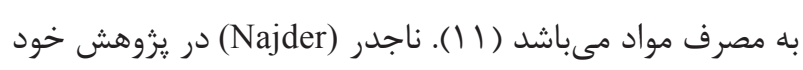

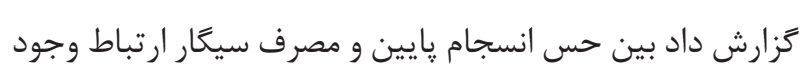
دارد (T) (T). در يزوهشى كه توسط كرينوستين (Grevenstein)

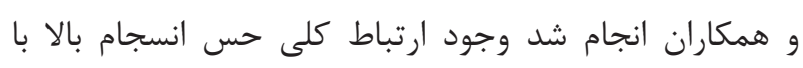

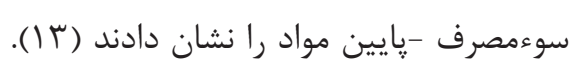

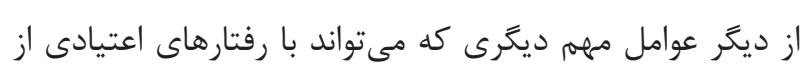

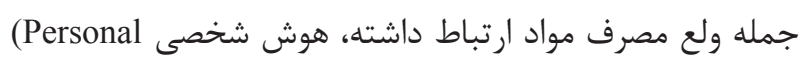
(Gardner) ميتقاد داشت كه هوش (intelligence)

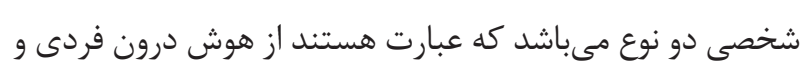

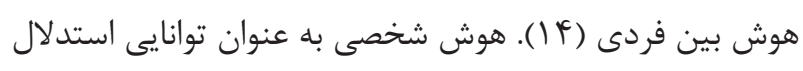



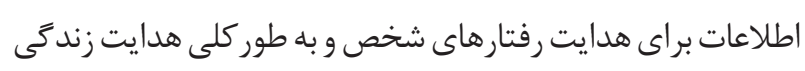
تعر يف شده است (له ). هوش شخصى، تركيبى از هوش هيجانى و هوش اجتماعى مى باشد كه در موضوعات اساسى نظير شخصيت، 
و معنى دارى Tr/A • گزارش كردند (YV). در ايران محمدىزاده و

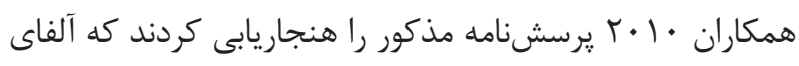

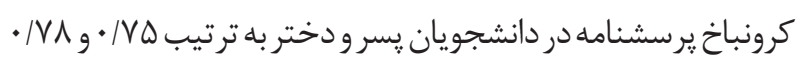

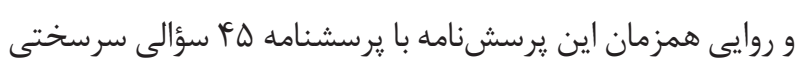

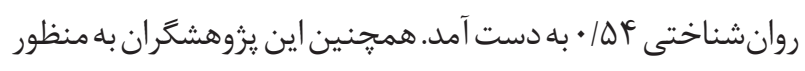

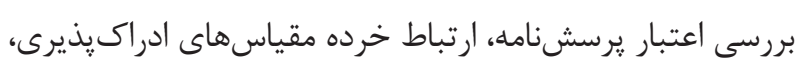

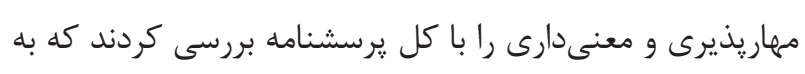

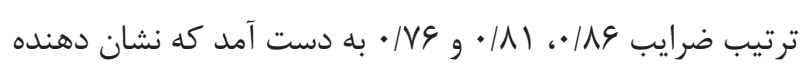

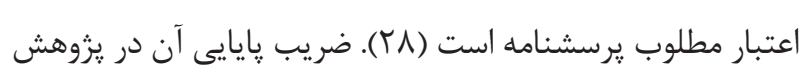

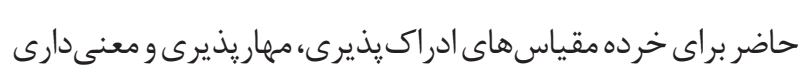

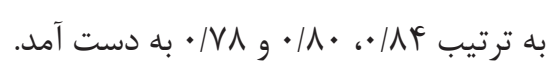

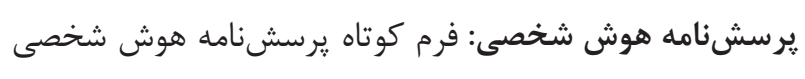

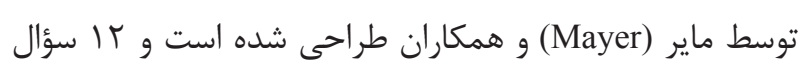

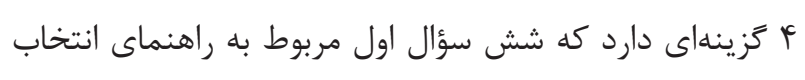

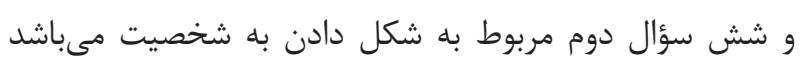

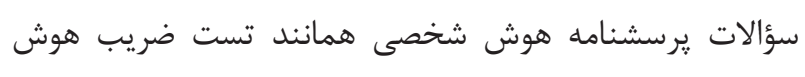

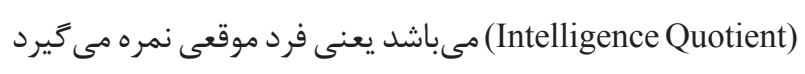

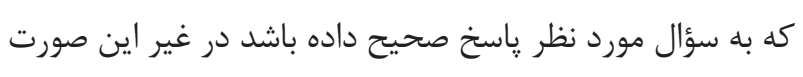

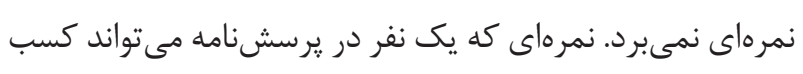

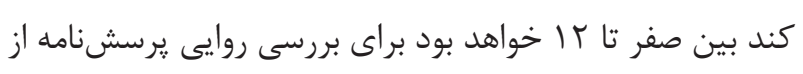

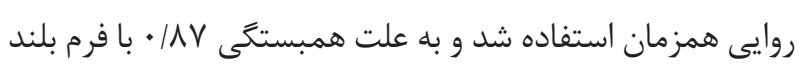

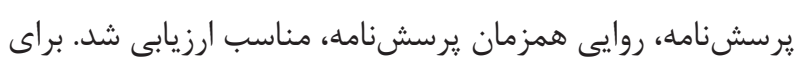



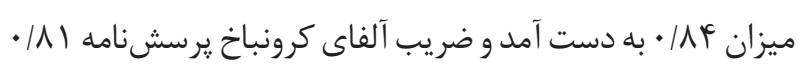

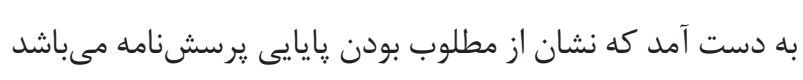

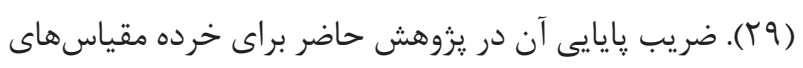

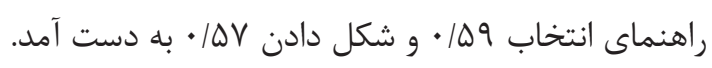
به منظور رعايت ملاحظات اخلاقى، وِ از از اخذ موافقت آكاهانه

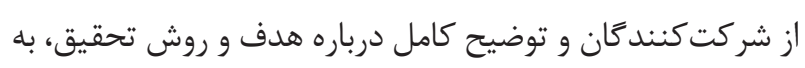
آنها اطمينان داده شد كه اطلاعات آنان محرمانه باقى خواهد ماند ماند. همجنين قوانين كميته اخلاق نشر رعايت كرديد. جهت تحليل داندان

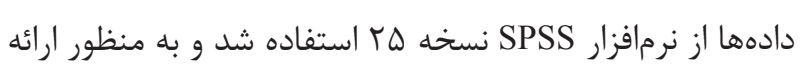

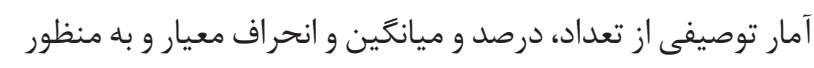

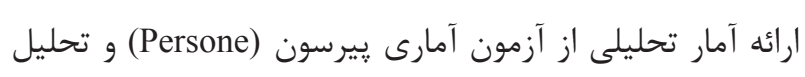

كه 19 مركز را شامل مىشد تهيه، سيس دو مركز از بين آنها به تصادف انتخاب و يس از مراجعه به مراكز مربوطه تمامى يرونده مهاى

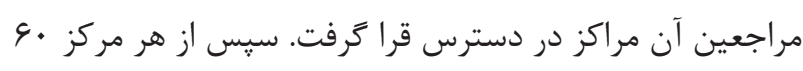

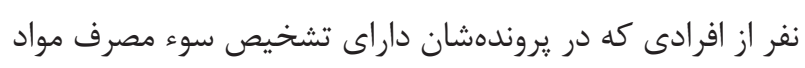



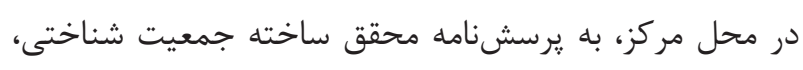

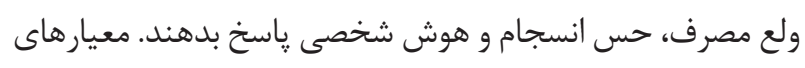

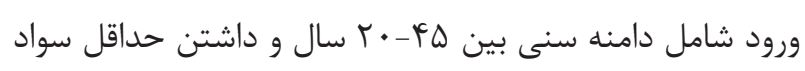

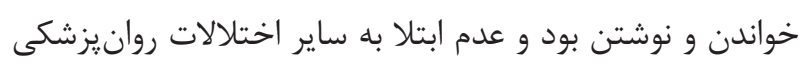

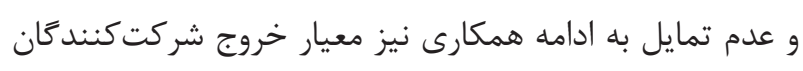
بود. ابزارهاى تردآورى دادهها شامل موارد زير بودهاند:

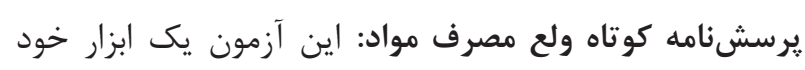
كزارشى ^ آيتمى است كه توسط سوموزا (Somoza) و همكاران

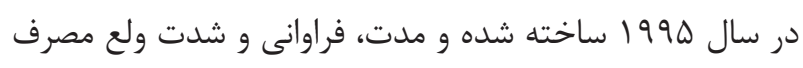



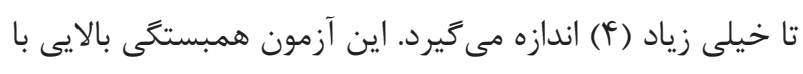

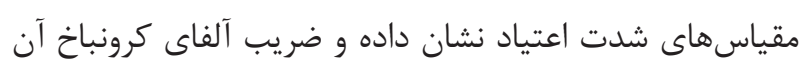

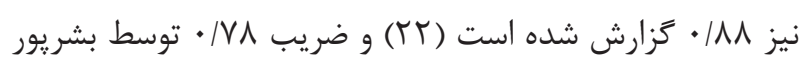
(Basharpoor) يزوهش حاضر • • • • به دست آمد. يرسشنامه حس انسجام: نسخه كوتاه يرسشنامه حس انسجام

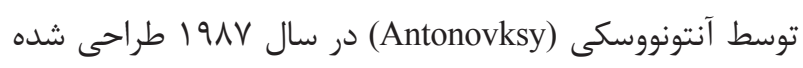

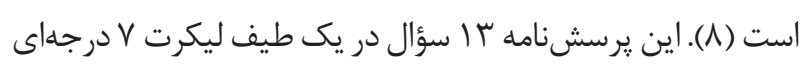

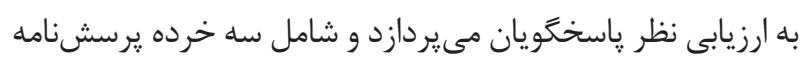

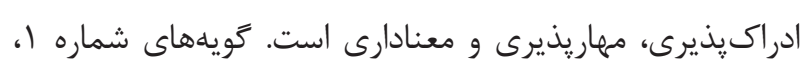



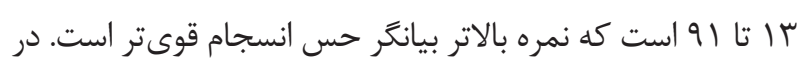

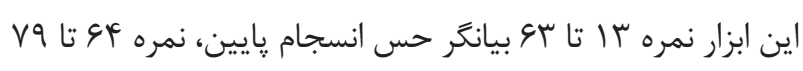

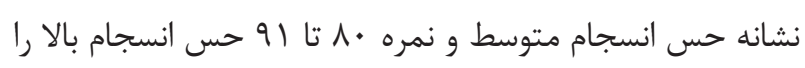

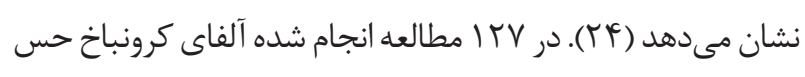

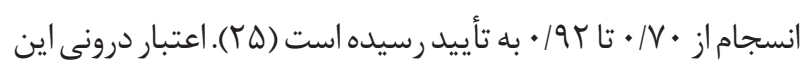

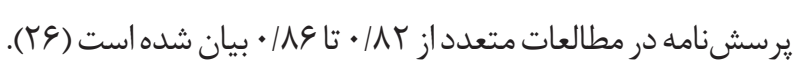

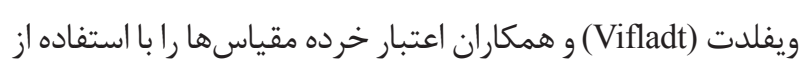

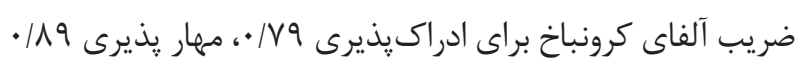


واتسون (Durbin-Watson) (قراركيرى در بازهى 1/ه تا ه/هآ)

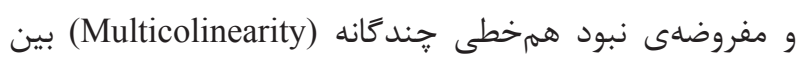
متغيرهاى مستقل با استفاده از شاخص تولرانس (Tolerance)

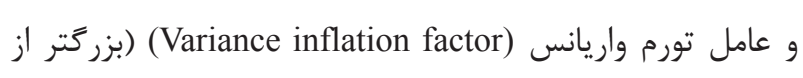
1/·)، مورد تأييد قرار ترفت.



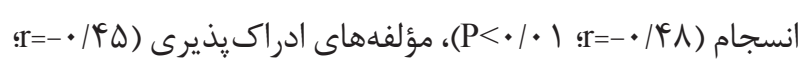
؛

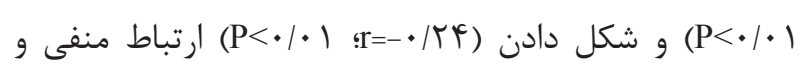
معنادار دارد.

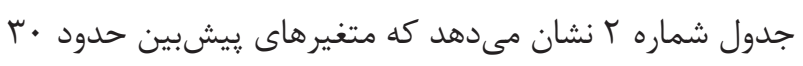

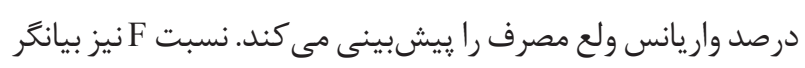

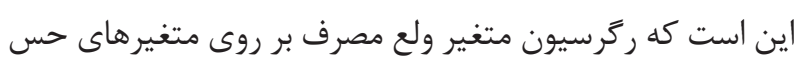

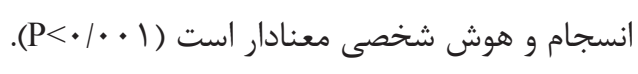

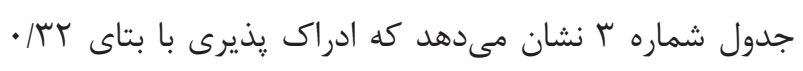

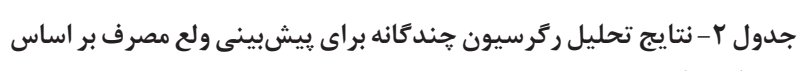
حس انسجام و هوش شخصى

\begin{tabular}{cccc}
\hline of FSig & $\mathbf{F}$ & $\mathbf{R}^{r}$ & $\mathbf{R}$ \\
\hline$\cdot / \cdot .1$ & $9 / \Delta \mathrm{Fr}^{\mathrm{r}}$ & $\cdot / \mathrm{r} \cdot$ & $\cdot / \mathrm{QFT}$ \\
\hline
\end{tabular}

ركرسيون (Regresion) جند رًانه استفاده گرديد.

\section{يافتهها}

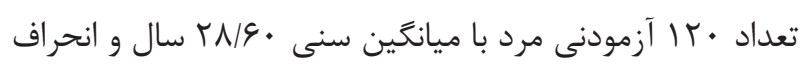

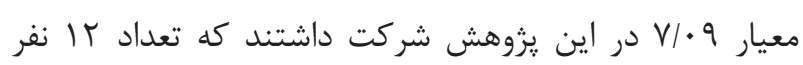

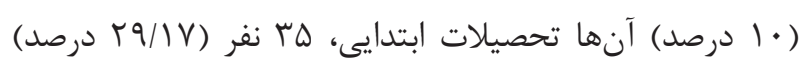

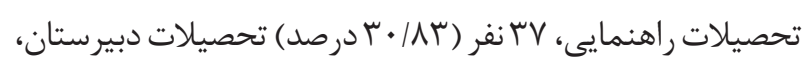

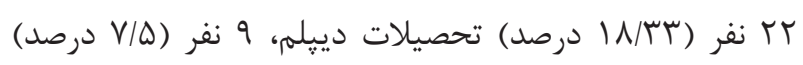

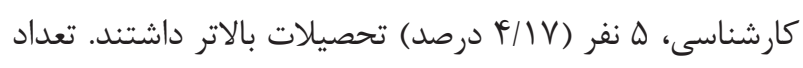

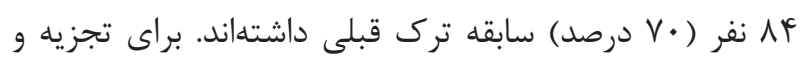
تحليل دادهها از ضريب همبستخى بيرسون (Person) و ركرسيون

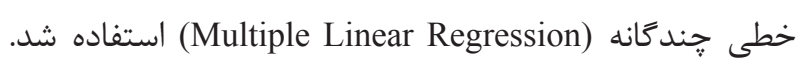

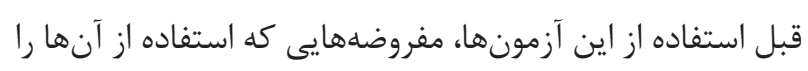
مجاز مىشمارند، مورد بررسى قرار گرفت. فرضيه وجود رابطهى إنى

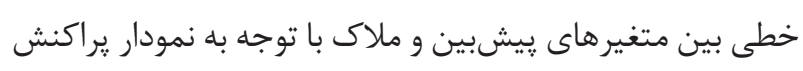

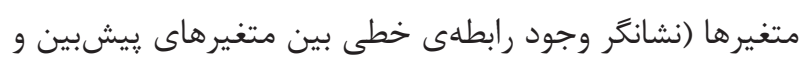
ملاك)، مفروضهى نرمال بودن توزيع دادهها با استفاده از آزمون



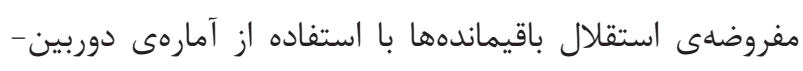

\begin{tabular}{|c|c|c|c|c|c|c|c|c|}
\hline ( () & (द) & $(\Delta)$ & $(\boldsymbol{F})$ & $(\boldsymbol{r})$ & $(Y)$ & (1) & $\mathbf{M} \pm \mathbf{S D}$ & متغير \\
\hline & & & & & & 1 & $G 1 \cdot \varphi \pm r r / \cdot V$ & حس انسجام (1) \\
\hline & & & & & 1 & $\cdot 1 \Lambda \cdot * * *$ & $r / \Delta \Delta \pm \Lambda / I V$ & ادراك يذيرى (T) \\
\hline & & & & 1 & $\Delta r^{*}{ }^{*}$ & $\cdot 19 \cdot * * *$ & $r / \Delta १ \pm V / ৭ \vee$ & مهار يذيرى (ז) \\
\hline & & & 1 & $\cdot / V \Lambda^{* * *}$ & $\cdot / 49^{* * *}$ & $\cdot / \wedge \Delta^{* * * *}$ & $r / \cdot r \pm V / q r$ & معنادارى (F) \\
\hline & & 1 & $\cdot 1 \cdot \mathrm{V} \cdot$ & $\cdot 1 \cdot \cdot 1$ & $\cdot 1 \cdot \mathrm{i} \wedge$ & $\cdot 1 \cdot 9$ & $1 / \Delta \pm \pi / T r$ & راهنماى انتخاب (ه) \\
\hline & 1 & $\cdot / K \Psi^{* * *}$ & .114 & $\cdot 1 \cdot 49$ & $\cdot 1 \cdot 14$ & (1. & $1 / \cdot \varphi \pm Y / 4 \cdot$ & شكل دادن (ع) \\
\hline 1 & $-\cdot / T Y^{* * *}$ & $-\cdot / 11$ & $-\cdot / r V^{* * *}$ & $-\cdot / \mathbb{f} \cdot * *$ & $-\cdot / \mathbb{F} \Delta^{* * *}$ & $-\cdot / \mathcal{C} \Lambda^{* * * *}$ & T/FF士IV/Tr & ولع مصرف (V) \\
\hline
\end{tabular}

\begin{tabular}{|c|c|c|c|c|c|c|}
\hline $\mathbf{P}$ & $\mathbf{t}$ & $\boldsymbol{\beta}$ & STE & B & متغيرهاى بيشبين & \\
\hline$\cdot 1 \cdot \cdot 1$ & $9 / 11$ & & $1 / / F$ & 1.199 & مقدار ثابت & \multirow{4}{*}{ ولع مصرف } \\
\hline$\cdot 1 \cdot \cdot 1$ & 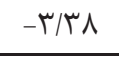 & - & $.11 \mathrm{KG}$ & $-\cdot / 4 T G$ & ادراك يذيرى & \\
\hline$\cdot 1 \cdot \Delta$ & $-r / \cdot G$ & $-\cdot$ TFF & • & -.1199 & مهاريذيرى & \\
\hline$\cdot / \mathbb{f}$ & -•|Ars & $-\cdot 11 \cdot V$ & $\cdot / T 10$ & $-\cdot / 1 \wedge \cdot$ & معنادارى & \\
\hline
\end{tabular}


جدول F - ضرايب بتا و آزمون معنادارى تى بر اساس مؤلفههاى هوش شخصى

\begin{tabular}{|c|c|c|c|c|c|c|}
\hline $\mathbf{P}$ & $\mathbf{t}$ & $\beta$ & STE & بتا & متغير هاى پِيشبين & \\
\hline$\cdot 1 \cdot \cdot 1$ & $\mid f / T s$ & & $1 / \cdot 9$ & $\mid \Delta / \cdot \cdot$ & مقدار ثابت & \multirow{3}{*}{ ولع مصرف } \\
\hline$\cdot 1 \cdot \Delta$ & $-Y / Y Y$. & $-\cdot|r| F$ & $\cdot / r \cdot$ & $-\cdot|9 V|$ & شكل دادن & \\
\hline.$|0|$ & • & .1 .94 & $\cdot / M F$ & $\cdot / 499$ & راهنماى انتخاب & \\
\hline
\end{tabular}

مورد مواجهه آنها در آينده پيشبينيذير باشند يا حداقل وقتى با رويداد ناگمهانى مواجهه شدند رفتارى واضح و مرتبط از خود نشان

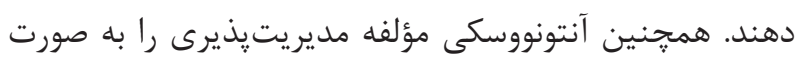

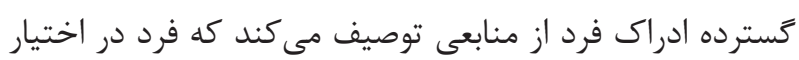

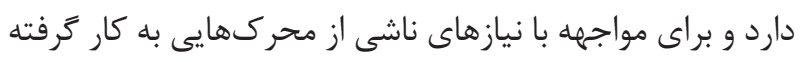

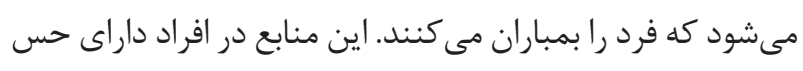
انسجام، بسنده و مؤثر هستند و نيز معنادارى يك مؤلفه كاملاً

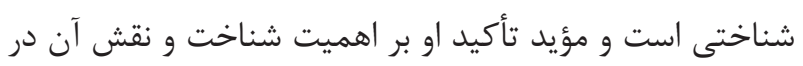
حس انسجام است و آنتونووسكى معتقد است كه معنادارى، مؤلفه انكَيزشى حس انسجام است، زيرا همين مؤلفه است كه فرد را به اسه طرف ارتقاء فهمم از دنياى شخص و منابع تحت اختيار سوق مى دهدي

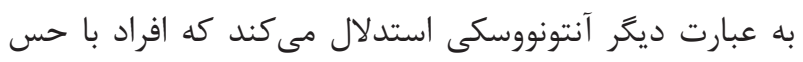

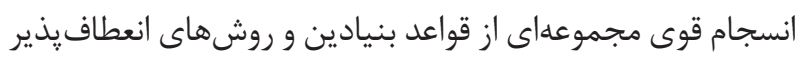

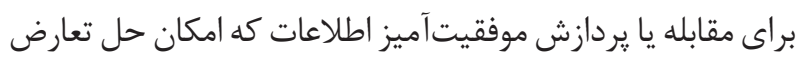

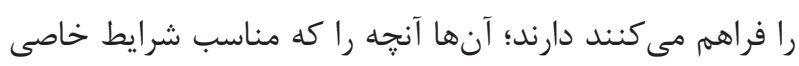
است را انجام مىدهند. در اين مطالعه نشان داده شد كه افراد با فيا

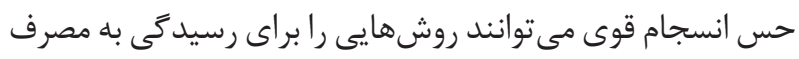

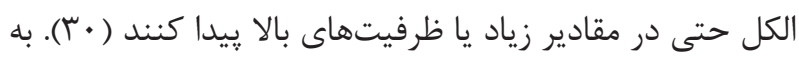

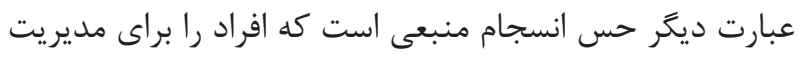

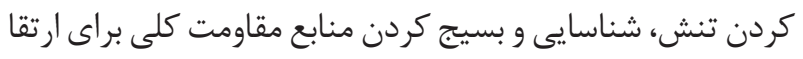

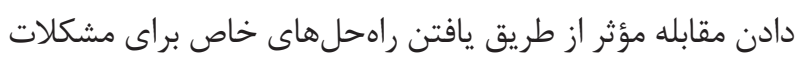

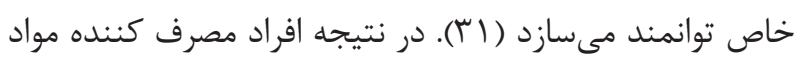

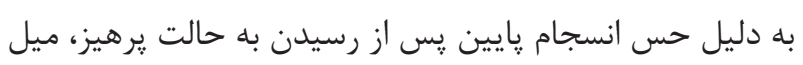

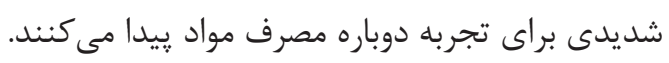

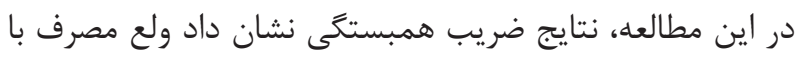

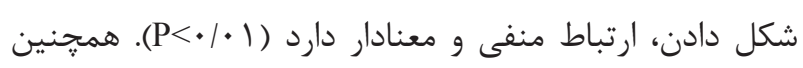

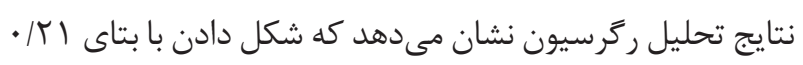

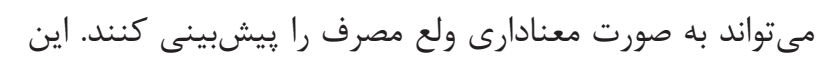

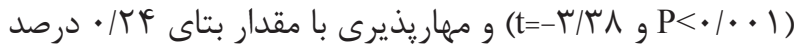
مى توانند به صورت معنادارى ولع مصرف را بيشبينى كنند.

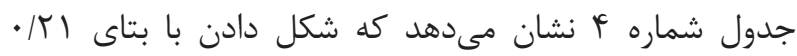

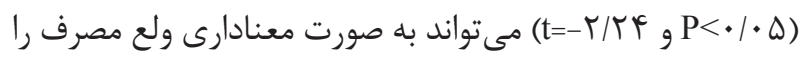

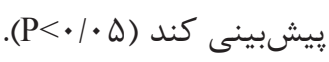

\section{بحث و نتيجه كيرى}

يزوهش حاضر با هدف نقش حس انسجام و هوش شخصى در

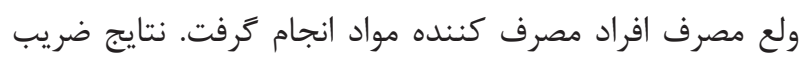
همبستخى نشان داد ولع مصرف با نمره كل حس انسجام، مؤلفههاى مصري

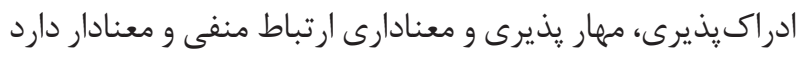

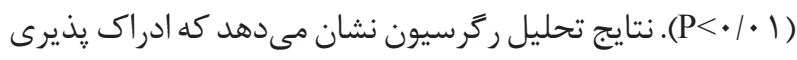

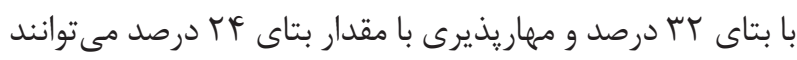

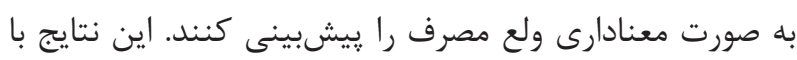
يافتههاى بورجخَريوينك (Borchgrevink) (••)، ناجدر (Najder)

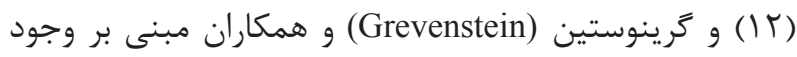
ارتباط كلى حس انسجام بالا با سوء مصرف پايين مواد همخوان مىباشد (1) (1). در تبيين اين فرض مىتوان كفت آنتونووسكى (Antonovksy)

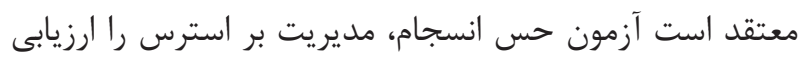

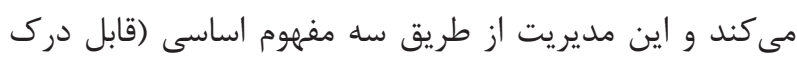

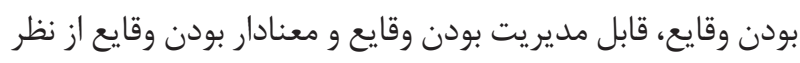
فرد) مفاهيم داراى جنبه روانى اجتماعى ممكن مى كند. قابليت

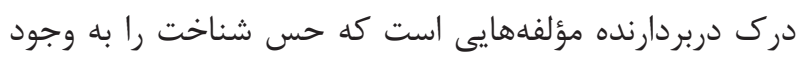

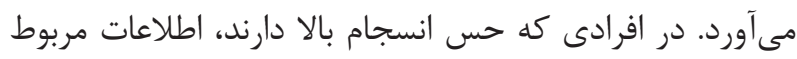

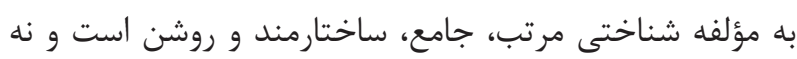

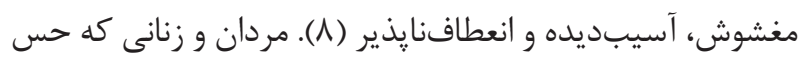

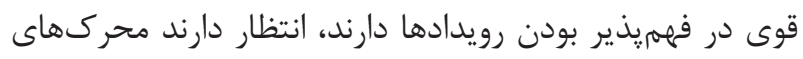




$$
\text { استفاده از ماده مصرفى و ميل شديد به آن مىشود. }
$$

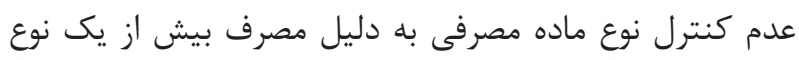

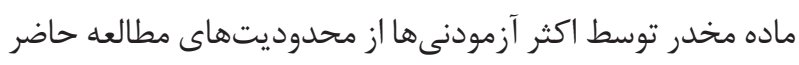

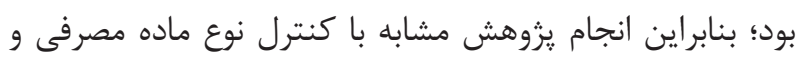

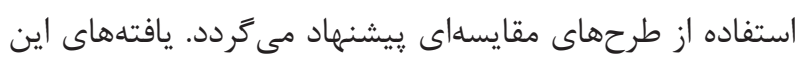

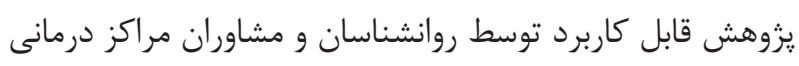

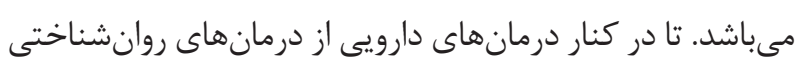

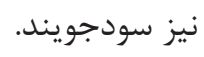

$$
\text { تشكر و قدردانى }
$$

بدين وسيله از مديريت، يرسنل و كليه درمانجويان مراكز ترى ولى اعتياد استان كرمانشاه به خاطر همكارى ارزندهشان در اجراى اين ورين

$$
\text { يزوهش، نهايت قدردانى را داريم. }
$$

تضاد منافع

بدين وسيله نويسندگان تصريح مى كنند كه هيج گَونه تضاد منافعى در خصوص يزوهش حاضر وجود ندارد.

\section{References}

1- Miller P. Principles of addiction: comprehensive addictive behaviors and disorders: Academic Press; 2013.

2- Uceyler N, Burgmer M, Friedel E, Greiner W, Petzke F, Sarholz $\mathrm{M}$, et al. [Etiology and pathophysiology of fibromyalgia syndrome : Updated guidelines 2017, overview of systematic review articles and overview of studies on small fiber neuropathy in FMS subgroups]. Schmerz. 2017;31(3):239-45. http://dx.doi. org/10.1007/s00482-017-0202-5 www.ncbi.nlm.nih.gov/ pubmed/28493226

3- Ahrnsbrak R, Bose J, Hedden SL, Lipari RN, Park-Lee E. Key substance use and mental health indicators in the United States: Results from the 2016 National Survey on Drug Use and Health. Center for Behavioral Health Statistics and Quality, Substance Abuse and Mental Health Services Administration: Rockville, MD, USA. 2017.

4- Kober H, Bolling D. Emotion regulation in substance use disorders. Handbook of emotion regulation. 22014. p. 428-46.

5- Auriacombe M, Dubernet J, Sarram S, Daulouède J, Fatséas M. Traitements pharmacologiques dans les addictions: pour une approche transversale et simplifiée. Traité d'addictologie: Lavoisier Paris; 2016. p. 307-10.
(IV) (Dumitrescua) ) رايس جويان و همكاران (19) و احمدى (1)

$$
\text { و همكاران (1) (1) همسو مىباشد. }
$$

در تبيين اين فرض مىتوان كفت افرادى كه هوش شخصى بالايى دارند با توجه به شناختى كه از شخصيت خود دارند سعى مى كنند خودشان را همانطور كه هستند قبول داشته باشند. هر نقص و كمبودى كه داشته باشند، باز هم خودشان را ارزشمند مىدانند و خود مفهومى آنها خيلى واقعى و منطقى مىباشد. بين خود واقعى و خودآرمانى آنها تفاوت زيادى وجود ندارد. اين حس باعث مىشود كه عزتنفس بالايى داشته باشند كه اين هم به نوبه خود باعث مىشود كه روابط آنها با ديخران بهبود ييدا كند و به راحتى بـى با اطرافيان رابطه برقرار كنند و ارتباط بهترى با اطرافيان داشته باشند (1))؛ به عبارت ديخر هوش شخصى يك توانايى ذهنى است كه موقع استدلال در مورد شخصيت و موضوعات مرتبط با بـ شخصيت نظير علت رفتار، نوع شخصيت، نوع اهداف و ارزشها مورد استفاده قرار مى گيرد و از آنجا كه در افراد مصرفكننده مواد، هوش شخصى يايين است اين افراد قادر به ارزيابى مشكل خود نيستند در نتيجه اين ناتوانى منجر به بروز نشانها و تحولات رفتارى در فرد مىشود. از جمله اين تحولات عدم كنترل بر روى

6- Serre F, Fatseas M, Swendsen J, Auriacombe M. Ecological momentary assessment in the investigation of craving and substance use in daily life: a systematic review. Drug Alcohol Depend. 2015;148:1-20. http://dx.doi. org/10.1016/j.drugalcdep.2014.12.024 www.ncbi.nlm.nih.gov/ pubmed $/ 25637078$

7- Bresin K, Verona E. Craving and substance use: Examining psychophysiological and behavioral moderators. Inter $\mathrm{J}$ Psychophysiology. 2019;3(18):1-12. http://dx.doi.org/10.1016/j. ijpsycho.2019.03.006

8- Antonovsky A. Unraveling the mystery of health: How people manage stress and stay well. San Francisco: Jossey-bass; 1987.

9- Bengtsson-Tops A, Hansson L. The validity of Antonovsky's Sense of Coherence measure in a sample of schizophrenic patients living in the community. J Adv Nurs. 2001;33(4):432-8. http:// dx.doi.org/10.1046/j.1365-2648.2001.01692.x www.ncbi.nlm.nih. gov/pubmed/11251730

10- Borchgrevink C. Predicting Alcohol Consumption in Hospitality Populations Using Sense of Coherence. J Tourism \& Hospitality Management. 2017;5(1). http://dx.doi.org/10.17265/2328$2169 / 2017.02 .003$ 
11- Arghabaei M, Soleimanian A, Mohammadipour M. The Role of Family Emotional Atmosphere, Sense of Coherence, and Affects in the Prediction of Tendency Toward Substance Use Among University Students. Iranian J Psychiatry \& Clinical Psychology. 2018. http://dx.doi.org/10.32598/ijpcp.24.3.310

12- Najder A. Sense of Coherence, Smoking Status, Biochemical Cardiovascular Risk Factors and Body Mass in Blue Collar Workers-Short Report. Am J Mens Health. 2018;12(4):894-9. http://dx.doi.org/10.1177/1557988317748393 www.ncbi.nlm.nih. gov/pubmed/29313407

13- Grevenstein D, Bluemke M, Nagy E, Wippermann C, KroeningerJungaberle H. Sense of coherence and substance use: Examining mutual influences. Personality and Individual Differences. 2014;64:52-7. http://dx.doi.org/10.1016/j.paid.2014.02.017

14- Mayer JD, Panter AT, Caruso DR. Does personal intelligence exist? Evidence from a new ability-based measure. J Pers Assess. 2012;94(2):124-40. http://dx.doi.org/10.1080/00223891.2011.646 108 www.ncbi.nlm.nih.gov/pubmed/22339306

15- Allen JL. Personal Intelligence and Learning about Personality in Everyday Life. 2017.

16- Mayer JD, Salovey P, Caruso DR. TARGET ARTICLES: "Emotional Intelligence: Theory, Findings, and Implications". Psychological Inquiry. 2004;15(3):197-215. http://dx.doi. org/10.1207/s15327965pli1503_02

17- Dumitrescu AL, Badițã D, Dogaru CB, Toma C, Duțã C. The Association of Social Desirability and Social Intelligence with Smoking among Undergraduates. Procedia - Social and Behavioral Sciences. 2014;159:552-6. http://dx.doi.org/10.1016/j. sbspro.2014.12.423

18- Ahmadi S, Yaghouti H, Fallahi V. The role of ethical enthusiasm and social intelligence in predicting the craving of drug users. J of Research in Addiction Research. 2017;11(44):155-68.

19- Raisjouyan Z, Talebi M, Ghasimi Shahgaldi F, Abdollahian E. Investigating the effect of emotional intelligence on the addiction relapse after quitting. Asia Pacific J Med Toxicology. 2014;3(1):27-30.

20- Luminet O, Cordovil de Sousa Uva M, Fantini C, de Timary P. The association between depression and craving in alcohol dependency is moderated by gender and by alexithymia factors. Psychiatry Res. 2016;239:28-38. http://dx.doi.org/10.1016/j.psychres.2016.02.062 www.ncbi.nlm.nih.gov/pubmed/27137959

21- Delavar A. Theoretical and scientific resources of research in humanities and social sciences. Tehran: Roshd publication; 2006.

22- Somoza E, Dyrenforth S, Goldsmith J, Mezinskis J, Cohen M, editors. In search of a universal drug craving scale. annual meeting of the American Psychiatric Association, Miami, FL; 1995.

23- Basharpoor S. Relationships between cognitive emotion regulation and effortful control with severity of dependence and craving in people with substance dependency. $\mathrm{J}$ of Substance Abuse Addiction Research. 2014;7(28):131-46.

24- Antonovsky A. The structure and properties of the sense of coherence scale. Social Science \& Medicine. 1993;36(6):725-33. http://dx.doi.org/10.1016/0277-9536(93)90033-z

25- Rohani C, Khanjari S, Abedi HA, Oskouie F, Langius-Eklof A. Health index, sense of coherence scale, brief religious coping scale and spiritual perspective scale: psychometric properties. J Adv Nurs. 2010;66(12):2796-806. http://dx.doi.org/10.1111/j.13652648.2010.05409.x www.ncbi.nlm.nih.gov/pubmed/20722813

26- Lustig DC, Rosenthal DA, Strauser DR, Haynes K. The Relationship Between Sense of Coherence and Adjustment in Persons with Disabilities. Rehabilitation Counseling Bulletin. 2016;43(3):13441. http://dx.doi.org/10.1177/003435520004300302

27- Vifladt A, Simonsen BO, Lydersen S, Farup PG. The association between patient safety culture and burnout and sense of coherence: A cross-sectional study in restructured and not restructured intensive care units. Intensive Crit Care Nurs. 2016;36:26-34. http://dx.doi.org/10.1016/j.iccn.2016.03.004 www.ncbi.nlm.nih. gov/pubmed/27212614

28- Mahammadzadeh A, Poursharifi H, Alipour A. Validation of Sense of Coherence (SOC) 13-item scale in Iranian sample. Procedia Social and Behavioral Sciences. 2010;5:1451-5. http://dx.doi. org/10.1016/j.sbspro.2010.07.306

29- Mayer JD, Allen JL. A Personality Framework for the Unification of Psychology. Review of General Psychology. 2013;17(2):196202. http://dx.doi.org/10.1037/a0032934

30- Nilsson KW, Starrin B, Simonsson B, Leppert J. Alcohol-related problems among adolescents and the role of a sense of coherence. Inter J Social Welfare. 2007;16(2):159-67. http://dx.doi. org/10.1111/j.1468-2397.2006.00452.x

31- Eriksson M, Lindstrom B, Lilja J. A sense of coherence and health. Salutogenesis in a societal context: Aland, a special case? J Epidemiol Community Health. 2007;61(8):684-8. http:// dx.doi.org/10.1136/jech.2006.047498 www.ncbi.nlm.nih.gov/ pubmed/17630366 